\title{
INFLUENCIA DE LA TECNOLOGÍA DE LA INFORMACIÓN EN EL ROL DEL PROFESORADO Y EN LOS PROCESOS DE ENSEÑANZA- APRENDIZAJE
}

\author{
(THE INFLUENCE OF THE INFORMATION TECHNOLOGY ON THE ROLE OF TEACHERS AND ON \\ THE TEACHING-LEARNING PROCESS)
}

\author{
Eloy López Meneses \\ María Jesús Miranda Velasco \\ Universidad de Extremadura (España)
}

\section{RESUMEN}

En este artículo reflexionamos sobre la importancia de la formación de los profesores, en un contexto sociocultural en el que Internet está entrando progresivamente en la vida cotidiana como un instrumento fundamental que afecta a los estudios, al trabajo, a la comunicación, transformándose en parte de nuestra cultura.

Las implicaciones de este hecho en los procesos de enseñanza aprendizaje, creemos que afectan, por un lado, a un saber vinculado con los profundos cambios económicos y sociales en marcha, con las nuevas tecnologías, con la nueva organización industrial e institucional. Por otro, se requiere una nueva actitud, y el desarrollo de nuevas capacidades en los docentes y en los discentes.

La utilización de las Nuevas Tecnologías de la Información y la Comunicación, la gestión de los nuevos entornos de aprendizaje, y el cambio metodológico, necesariamente implican un cambio de mentalidad, de prácticas docentes, y de nuevos roles del profesorado.

Palabras clave: nuevas tecnologías de la información y la comunicación, formación del profesorado, procesos de enseñanza-aprendizaje, Internet, educación.

\begin{abstract}
In this article we think about the importance of training teachers in a socio-cultural context where the Internet is progressively influencing our daily lives as a fundamental instrument that affects our studies, jobs, communication, becoming part of our culture.
\end{abstract}


We truly believe that the implications of this fact in the teaching-learning processes affect, on the one hand, the knowledge linked to the deep economic and social changes today, to the new technologies, to the industrial and institutional organisation; On the other hand, a new attitude is required as well as the development of new capacities in the teachers and students.

The use of New Information and Communication Technologies, the management of the new environment related to learning processes and the methodological change, necessarily imply a change in mentality, in teaching practices and new roles in the teaching staff.

Key words: new information and communication technologies, teaching staff training, teaching-learning processes, the internet, education.

Tal como señala Barry Wellman (2004) ${ }^{1}$ en The Internet in Everyday Life: An Introduction, Internet está afectando a las formas tradicionales de sociabilidad; cada vez más los usuarios pasan más tiempo navegando, utilizan más el e-mail, adquieren objetos con el comercio electrónico, hay más grupos de discusión; en síntesis, Internet está alcanzando mayores niveles de democratización e ingresa paulatinamente a la vida doméstica como un instrumento fundamental que afecta positivamente a los estudios, al trabajo, a la comunicación, siendo así parte de la cultura cotidiana.

En la misma línea, establecida en el argumento anterior, la Comisión Web-based Education, del año 2000, declara que "las transformaciones que la sociedad está viviendo en la última década, están penetrando y modificando el tejido y las bases de nuestra sociedad desarrollada”. Y, evidentemente, a estos cambios radicales están contribuyendo de forma manifiesta las tecnologías avanzadas de la información y la comunicación, y más particularmente Internet, que vienen afectando a todos los ámbitos de desarrollo y progreso social ${ }^{2}$.

Coincidimos con la experta en tecnología educativa, Luisa Torres (2005) que la sociedad ha cambiado considerablemente en un corto periodo de tiempo y continúa haciéndolo apresuradamente. Estos cambios no sólo se apoyan en un aumento cuantitativo de la información disponible por el avance de las nuevas tecnologías y en la inmediatez con que dicha información puede llegar a los destinatarios, sino que estimamos que están directamente relacionados con todas las modificaciones culturales, es decir, cambios en la forma de comunicarnos, de trabajar, de divertirnos, de aprender...

Como expusimos recientemente, en una publicación internacional (Cabero, Ballesteros; López Meneses, 2004), Internet se va convirtiendo, inexorablemente, en una especie de tejido nervioso que se va desarrollando rápidamente en nuestras 
sociedades y se perfila como una herramienta universal para el profesorado de Educación Secundaria en la búsqueda, el intercambio de información, las experiencias formativas y la investigación.

De lo expuesto, anteriormente, se puede plantear una serie de hechos significativos, como señalan Tiffin y Rajasinghan que "la escuela no funciona como antes" (1997, p. 101). En todo el mundo, las sociedades se enfrentan con un dilema de que sus sistemas de educación se diseñaron para satisfacer las necesidades de sociedades agrícolas e industriales, no de la futura sociedad de la información. Los sistemas educativos están preparando a las personas para el pasado, para las ideas, actitudes y valores de una forma de vida que se está desvaneciendo y para el trabajo en áreas donde la demanda de mano de obra es cada vez menor.

En consecuencia, creemos que llegado a este punto, con el inexorable transcurrir del tiempo, uno comienza a entrever, de una forma incipiente, e incluso algunas veces primitiva, la complejidad que subyace en el momento histórico que nos ha tocado vivir... un espacio temporal caracterizado por el descubrimiento de una plétora de medios de comunicación de masas (cine, prensa, radio, televisión digital, videojuegos online, teléfonos móviles con cámara digital y grabador/hologramas, reproductores y grabadores de imágenes digitales y la gran estrella, la red Internet) que conviven en las formas de comunicarnos y en los estilos y modos de consumir nuestro tiempo de ocio, y por supuesto, en la aldea escolar y en sus habitantes principales el profesor de Educación Secundaria y el alumnado.

Podríamos reseñar, a tenor de lo expuesto y a modo de conclusión, las palabras pronunciadas en la Universitat Oberta de Catalunya (UOC), por el profesor Manuel Castells $(2001)^{3}$, sobre el fenómeno de esta red de redes "Internet es la sociedad, expresa los procesos sociales, los intereses sociales, los valores sociales, las instituciones sociales [...], Internet es el corazón de un nuevo paradigma sociotécnico que constituye en realidad la base material de nuestras vidas y de nuestras formas de relación, de trabajo y de comunicación. Lo que hace Internet es procesar la virtualidad y transformarla en nuestra realidad, constituyendo la sociedad red, que es la sociedad en que vivimos."

\section{EL PROFESORADO DE EDUCACIÓN SECUNDARIA EN LA SOCIEDAD DE LA INFORMACIÓN Y EL CONOCIMIENTO}

Como expusimos en el Congreso Internacional Edutec 2004, ya no basta con saber, sino que también es necesario un saber vinculado con los profundos 
cambios económicos y sociales en marcha, con las nuevas tecnologías, con la nueva organización industrial e institucional, en un mundo crecientemente complejo e interdependiente, que requiere personas con viva inquietud creativa e innovadora, con espíritu crítico, reflexivo y participativo (Ballesteros, López Meneses; Torres, 2004).

"La red está cambiando los hábitos, conceptos y costumbres, por esto los profesionales de la educación necesitan conocer este medio. Su conocimiento, análisis, reflexión sobre las potencialidades y consecuencias de su uso y abuso, permitirán al estudiante una mayor comprensión del cambio social y cultural en el que estamos inmersos" (Pérez, 1998, p.192).

Compartimos con Cabero (2000a), que no debe caber la menor duda que la introducción de Internet va a tener un fuerte impacto en la modificación de los estilos docentes de los profesores, pasando del tradicional suministrador de información a otros más novedosos: proveedor de recursos para los estudiantes no sólo en la asignación o recomendación al estudiante con los que deban de trabajar, sino lo que es más importante transformando los materiales para adaptarlos a las necesidades de los alumnos; organizador del aprendizaje, en el sentido de crear en el aula entornos específicos para su utilización y tiempo destinado para ello; tutor del estudiante; investigador; moderador y facilitador.

En coherencia con lo anterior, se desprende, tal como dice Mason (1998), que "la naturaleza de los nuevos entornos de aprendizaje se concentra, en una ruptura de la distinción entre profesor y alumno y se orientan hacia la construcción colectiva de la formación”.

Teniendo en cuenta todos los aspectos mencionados, anteriormente, coincidimos con Tascón (2003) que el aprovechamiento de las Nuevas Tecnologías de la Información y la Comunicación, la gestión de los nuevos entornos de aprendizaje y el cambio metodológico exige un cambio de mentalidad y de prácticas docentes, que además deben enfocarse no sólo en sentido instructivo sino también atendiendo a los aspectos educativos y de nuevos roles:

- Promotor de climas organizacionales; diseñador y gestor de actividades y entornos de aprendizaje que contemplen la diversidad de ritmos, estilos cognitivos, conocimientos y capacidades de los estudiantes.

- Orientador, guía de aprendizajes y del desarrollo de las capacidades de los alumnos (debe enseñar a aprender), asesor. 
- Motivador, provocador de curiosidad intelectual y entusiasmo, estimulador de aprendizajes, dinamizador de los grupos de trabajo colaborativo.

- Fuente de información (pero menos que en décadas anteriores) consultor que resuelve dudas.

- Promotor del uso de las TIC en diversos ambientes (biblioteca, aula, casa...).

- Transmisor de experiencias.

- Evaluador de recursos y proveedor de los mismos a los estudiantes.

- Creador de recursos (diseño y desarrollo).

- Co-aprendiz, con los estudiantes, promoviendo un descubrimiento guiado.

- Tutor.

- Investigador que reflexiona sobre la práctica y colabora con otros docentes.

- Actualizador de los contenidos de la asignatura, revisión de los planes de estudios y la bibliografía.

En la sociedad actual consideramos que los docentes, sin tener que ser grandes expertos en el manejo de tecnología, deberían ser usuarios en el dominio de las herramientas básicas que ofrecen los nuevos avances tecnológicos (manejo del navegador web, uso del correo electrónico, diseñadores de sitios web a nivel informativo, diseñadores de webquests, manejo del procesador de texto y de algún programa de tratamiento gráfico, entre otras herramientas), siendo estos utensilios informáticos, de una forma progresiva cada vez más motivadores, con entornos gráficos cada vez más intuitivos y amigables, caminado inexorablemente de una usabilidad rupestre hacia una usabilidad más amigable, intuitiva, afectiva ${ }^{4}$, e incluso la usabilidad emocional, emotional design ${ }^{5}$.

Por otra parte, estimamos que los profesionales de la educación deben ser, en la medida de sus posibilidades, constructores de gran parte de sus materiales hipermedias de enseñanza, evaluadores de los medios didácticos y de las estrategias de información seguidas por los estudiantes; así como, dinamizadores y organizadores de las posibles propuestas de actividades individuales y colectivas, orientadores; y, además de facilitadores y creadores de comunidades de conocimientos compartidos entre estudiantes.

Compartimos con el profesor Julio Cabero (200ob, 2003), que el rol del profesor deberá ser modificado de manera que frente al tradicional, transmisor de información, se impulsarán otras funciones, como son el de consultor de información, facilitadores de información; diseñadores de medios adaptados a las características de sus estudiantes y potencialidades de la tecnología utilizada; evaluadores continuos y asesores-orientadores. 


\section{REFLEXIONES FINALES}

Las tecnologías de comunicación digitales, y especialmente Internet, representan, en estos momentos, uno de los retos que tiene ante sí la Educación Superior, ya que permiten renovar sustantivamente los procesos formativos en múltiples formas y alterar, en consecuencia, las formas de comunicación entre docentes y alumnado, así como los procesos de enseñanza y aprendizaje. Todavía nos encontramos en una fase inicial de las aplicaciones de los ordenadores a la enseñanza superior, aunque, también es cierto que en estos últimos años se han dado pasos importantes. Gran parte de las universidades europeas, y entre ellas las españolas, disponen de recursos y espacios virtuales destinados a la docencia a través de Internet (Área y otros, 2003).

Como ha sintetizado Barberá "con estas nuevas tecnologías el docente [...] se convierte en un animador de la inteligencia colectiva de los grupos que se responsabiliza. Desde este punto de vista, su actuación se dirige al acompañamiento y gestión del aprendizaje: incitación al intercambio de conocimientos, mediación relacional y simbólica o al pilotaje personalizado de los recorridos de aprendizaje" (2001, p. 59).

En otro orden de cuestiones, como expusimos, en otro proyecto, Cabero, López Meneses; Ballesteros (2001) consideramos que los profesionales de la educación tienen el derecho y el deber de investigar sobre los enigmas de nuestra naturaleza y de la tecnología de nuestro contexto histórico actual, para orientar en la formación de sus alumnos y que sean capaces de relacionarse, interactuar, reflexionar críticamente con los nuevos medios. En este sentido, las nuevas tecnologías utilizadas racionalmente darán pie a una formación humana más flexible, coherente y autónoma (Loscertales, 2000).

Y otro elemento de reflexión muy importante, como plantean los expertos Jesús Salinas (1999) y Mercè Gisbert Cervera ${ }^{6}$, entre otros, que el profesorado docente necesita un proceso de formación y que la planificación del mismo y la misma existencia de formadores de formadores constituyen un tema clave. Pero, además, debemos pensar en términos de formación continua y de desarrollo profesional. El profesor de Educación Secundaria no sólo debe estar al día de los descubrimientos en su campo de estudio, debe atender al mismo tiempo a las posibles innovaciones en los procesos de enseñanza-aprendizaje y en las posibilidades de las tecnologías de la información y la comunicación. Deben prepararse para el nuevo rol de profesor como guía y facilitador de recursos que oriente a alumnos activos que participan en 
su propio proceso de aprendizaje; la gestión de un amplio rango de herramientas de información y comunicación actualmente disponibles y que pueden aumentar en el futuro las interacciones profesionales con otros profesores y especialistas de contenido dentro de su comunidad, pero también foráneos.

En este sentido, es de vital necesidad una educación acorde con una alfabetización que facilite a los estudiantes los mecanismos necesarios para interpretar de forma crítica el lenguaje de los medios, para descubrir sus mensajes, discriminando lo que hay en ellos de información y lo que hay de manipulación tergiversada de la realidad, hacia qué orientan, cuáles son los trasfondos que están detrás de los monopolios informativos y publicitarios; en definitiva, descubrir el "currículo oculto de las nuevas tecnologías” (Duarte, 2002). De forma que les permita desarrollarse, como ciudadanos activos, participativos y reflexivos ante los nuevos medios de comunicación de masas, así como desarrollar sus capacidades críticas para poder evaluar y valorar los mensajes verbo-icónicos que conviven constantemente con nosotros.

Podríamos decir, a tenor de lo expuesto y a modo de síntesis, que el profesor de Educación Secundaria del actual milenio, debería elaborar una programación de una forma flexible y muy bien estructurado, anticipándose a las posibles dudas de los alumnos, junto al establecimiento de rutas abiertas de comunicación e intercambio con los demás miembros de la comunidad educativa, vía web, para facilitar la creación de entornos formativos de Educación Secundaria, que promuevan la construcción del conocimiento adaptado a las necesidades del alumnado. Por otra parte, los profesionales de la Educación Secundaria deberían adoptar el rol de moderador, guía, orientador y tutor a sus discentes, y tratar, en la medida de lo posible, un reciclaje y una formación permanente en el ámbito de los recursos telemáticos. Además, si se nos permite el término de mentores que ilumine los proyectos futuros de nuestros educandos caminando hacia la calidad de la educación general del alumnado de la ESO.

\section{NOTAS}

1. Su dirección electrónica es: http://www.chass.utoronto.ca/ wellman/publications/ index.html

2. Su dirección electrónica es: http://www.ed.gov/offices/AC/WBEC/FinalReport/ WBECReport.pdf

3. Conferencia inaugural del curso académico 2001-2002 de la UOC. Su dirección electrónica: http://www.uoc.es/web/esp/launiversidad/inauguralo1/cultura.html

4. Compartimos con Yusef Hassan Montero y Francisco Jesús Martín Fernández (2003) que una interfaz, además de adaptarse, puede modelar los estados anímicos 
Influencia de la Tecnología de la Información en el Rol del Profesorado y en los Procesos...

del usuario, ya sea implícitamente (a través de la estética), o explícitamente, es decir, expresando afecto. Más información en: http://www.nosolousabilidad.com/ articulos/interfaces afectivas.htm

5. Como apunta la ingeniería Kansei: incorporar emoción y afecto en el proceso de diseño. Kansei viene a ser la definición de User Experience en Japonés: cómo percibe mentalmente un usuario un producto. También se define como Ingeniería Sensorial o Usabilidad Emocional. Mediante esta técnica se detectan aquellos atributos de un diseño que permiten la obtención de determinadas respuestas subjetivas por parte de las personas y diseñar basándonos en la persecución de esas respuestas. Para este método se utilizan objetos que permitan la obtención de respuestas extremas: agradable-desagradable; atractivo-feo; fácil de usar-complicado; sencillo-complejo. Su dirección electrónica: http://www.grancomo.com/glosario.php? $\mathrm{x}=\mathrm{K}$

6. Su dirección electrónica es: http://tecnologiaedu.us.es/edutec/2libroedutec99/libro/ total3.htm.

\section{REFERENCIAS BIBLIOGRÁFICAS}

Área, M. (2000). ¿Qué aporta Internet al cambio pedagógico en la Educación Superior?, en: Pérez, R. (Coord) Redes multimedia y diseños virtuales. Actas del III Congreso Internacional de Comunicación, Tecnología y Educación. Oviedo: Dpto. de Ciencias de la Educación de la Universidad de Oviedo, 128-135.

Área, M. y otros. (2003). Diseño y experimentación pedagógica de materiales didácticos distribuidos a través de la www. La web docente de la asignatura de tecnología educativa, en: Área, M. y Castro, J. J. (Coords). Actas de la I Jornadas Canarias sobre las tecnologías de la información y la comunicación en la docencia universitaria. Edullab. Las Palmas de Gran Canarias: Universidad de La Laguna, 283-298. [en línea]. Disponible en: http://www.edulab.ull. es/jornadas/actas/documentos/actas completas.pdf [consulta 2006, 20 de Junio]

Ballesteros, C.; López Meneses, E.; Torres, L. (2004). Las plataformas Virtuales: escenarios alternativos para la formación, en: I Congreso
Internacional sobre Educación $y$ Tecnologías de la Información y la Comunicación, Edutec 2004, Educar con tecnologías, de lo excepcional a lo cotidiano. [en línea]. Disponible en: http://edutec2004.lmi.ub.es/pdf/195. pdf [consulta 2006, 20 de Junio]

Barberá, E. y otros (2001). Enseñar y aprender a distancia: ¿es posible?. [en línea]. Disponible en: http://www. uoc.es/web/esp/art/uoc/0105018/ ensapren.html [consulta 2006, $20 \mathrm{de}$ Junio]

Cabero, J. (2000a). Las nuevas tecnologías y las transformaciones delas instituciones educativas, en: Lorenzo, M. y otros (Eds) Las organizaciones educativas en la sociedad neoliberal. Granada: Grupo Editorial Universitario, 463-493.

Cabero, J. (200ob). Las nuevas tecnologías al servicio del desarrollo de la Universidad: las teleuniversidades, en: Rosales, C. (coord.) Innovación en la Universidad. Santiago de Compostela, NINO, 187-216. [en línea]. Disponible en: $\quad$ http://tecnologiaedu.us.es/ bibliovir/pdf/86.pdf [consulta 2006, 20 de Junio]

Cabero, J.; López Meneses, E.; Ballesteros, 
C. (2001). La Asignatura de Nuevas Tecnologías aplicadas a la Educación: Un camino hacia la alfabetización tecnológica. Píxel-Bit. Revista de Medios y Educación, 17, 99-110. [en línea]. Disponible en: http://www.sav. us.es/pixelbit/articulos/n17/n17art/ art1710.htm [consulta 2006, 20 de Junio]

Cabero, J. (2003). La galaxia digital y la educación: los nuevos entornos de aprendizaje, en Aguaded, J. I.: Luces en el laberinto audiovisual, Huelva: Grupo Comunicar, 102- 121. [en línea]. Disponible en: http://tecnologiaedu. us.es/bibliovir/pdf/galaxia.pdf [consulta 2006, 20 de Junio]

Cabero, J.; Lopez Meneses, E. y otros. (2004). ¿Cómo mejorar la práctica profesional de los docentes universitarios? Algunos recursos y utilidades telemáticas. Píxel-Bit. Revista de Medios y Educación, 22, 5-23. [en línea]. Disponible en: http:// www.sav.us.es/pixelbit/articulos/ n22/n22art/art2202.htm [consulta 2006, 20 de Junio]

Castells, M. (2001). La cultura de libertad como constitutiva de Internet. Conferencia inaugural del curso académico 2001-2002 de la UOC [en línea]. Disponible en: http:// www.uoc.es/web/esp/launiversidad/ inauguralo1/cultura.html [consulta 2006, 20 de Junio]

Duarte, A. (2000). Los materiales hipermedias y multimedias aplicados, en: Cabero, J. (ed.): Nuevas Tecnologías aplicadas a la Educación. Madrid: Síntesis, 137-158.

Loscertales, F. (2000). El rol del profesor ante el impacto de las nuevas tecnologías, en: Cabero, J. (coord.) Las Nuevas Tecnologías para la mejora educativa. Sevilla: Kronos.

Mason, R. (1998). Models of Online Courses. ALN Magazine 2 (2). [en línea]. Disponible en: http://www. aln.org/publications/magazine/v2n2/ mason.asp [consulta 2006, 20 de Junio]

Pérez, A. (1998). Introducción a Internet, en: Cebrián, M.y otros (Coord) Creación de materiales para la Innovación Educativa con Nuevas Tecnologías. Málaga: I.C.E. Edutec `97.

Punset, E. (2000). Revolución de Internet. Programa televisivo de divulgación científica denominado REDES Programa emitido en la segunda cadena de RTVE, el 15 de octubre, número 174. [en línea]. Disponible en: http://www. rtve.es/tve/b/redes/semanal/prg174/ frsemana.htm [consulta 2006, 20 de Junio]

Tascón, C. (2003). De la formación a la teleformación: principios psicoinstruccionales, en: Área, M; Castro, J. J. (Coords) Actas de la I JornadasCanariassobrelastecnologías de la información y la comunicación en la docencia universitaria. Edullab. Las Palmas de Gran Canarias: Universidad de La Laguna, 39-47.

Tiffin, J.; Rajasingham, L. (1997). En busca de la clase virtual. La educación en la sociedad de la información. Barcelona: Paidós.

Torres, L. (2005). Elementos que deben contener las páginas web educativas. Píxel-Bit. Revista de Medios y Educación, 25. [en línea]. Disponible en: http://www.sav.us.es/pixelbit/ articulos/n25/n25art/art2508.htm [consulta 2006, 20 de Junio]

Web-Based Education Commission (2000). The power of the Internet for learning: moving from promise to practice. Moving from promise to practice. Report of the Web-Based Education Commission. Washington, D C. [en línea]. Disponible en: http:// www.ed.gov/offices/AC/WBEC/ FinalReport/WBECReport.pdf [consulta 2006, 20 de Junio] 


\section{PERFIL ACADÉMICO Y PROFESIONAL DE LOS AUTORES}

Eloy López Meneses, Profesor Colaborador (L.O.U.) en el Departamento de Ciencias de la Educación en la Facultad de Formación del Profesorado en Cáceres. Miembro del Grupo de Investigación Didáctica (G.I.D.): Análisis tecnológico y analítico de los procesos de enseñanza y aprendizaje desde el año 1999. Investigador en diferentes proyectos subvencionados relacionados con el uso de las redes de aprendizaje en la docencia de las universidades públicas españolas.

E-mail: eloylope@unex.es

María Jesús Miranda Velasco, Doctora por la Universidad de Salamanca. Profesora del Departamento de Ciencias de la Educación de la Universidad de Extremadura. Imparte docencia en Educación Social, Magisterio, y en el Programa de Doctorado. Profesora visitante del Programa Sócrates en el Instituto de Educación de Estocolmo, y en la Facultad de Formación del Profesorado de la Universidad de Bolonia. Investigadora en proyectos financiados en el contexto educativo del Espacio Europeo de Educación Superior.

E-mail:mirandav@unex.es

DIRECCIÓN DE LOS AUTORES

Universidad de Extremadura

Campus Universitario.

Avenida de la Universidad, s/n.

10071, Cáceres, España

Fecha de recepción del artículo: 04/12/06

Fecha de aceptación del artículo: 22/02/07 